\title{
HE4 and its evolving role in assessing tumor prognosis in gynecological and systemic malignancies
}

\author{
Shailendra Kapoor
}

Received: 12 February 2013/ Accepted: 26 February 2013/Published online: 15 March 2013

(C) Springer-Verlag Berlin Heidelberg 2013

To the Editor,

The recent article by Azzam et al. [1] provided for highly stimulating and interesting reading. HE4 may be helpful in assessing clinical prognosis in a number of other systemic malignancies.

HE4 may also be of prognostic value in patients with endometrial cancers. In fact, HE4 levels are closely reflective of the myometrium invasion levels in endometrial carcinomas [2]. When using $70 \mathrm{pmol} / \mathrm{l}$ as threshold, it has a sensitivity of about $59 \%$. Its negative predictive value is $71.5 \%$ [3]. HE4 levels bear a close association with overall survival in these patients. When used in conjunction with CA125, the overall specificity and sensitivity are markedly improved. Mutz-Dehbalaie et al. [4] have reported a hazard ratio of 4.04 for overall survival when the dual markers are used simultaneously. When used independently, its positive predictive value is $100 \%$ [5]. The diameter of the primary endometrial carcinoma is also closely co-related with HE4 levels.

HE4 is also of value in assessing clinical prognosis and outcome in individuals with lung adenocarcinomas. A 5-year survival rate of $97.1 \%$ has been noticed in patients negative for HE4 in contrast to a 5-year survival rate of $52.6 \%$ in those positive for HE4 [6]. In addition, malignant recurrence of the adenocarcinomas is closely reflected in serum HE4 levels. Nearly $89 \%$ of small cell lung cancer patients showed elevated HE4 levels [7]. In addition, the CEA status of the tumor bears a close positive relationship with the HE4 levels. Similarly, comparatively higher HE4 levels have been noted in malignant pleural effusions in contrast to lower levels in non-malignant effusions. This

S. Kapoor $(\square)$

Private Practice, Mechanicsville, VA, USA

e-mail: shailendrakapoor@yahoo.com test has a specificity of about $91 \%$. Nearly $90 \%$ of nonsmall cell lung carcinomas exhibit accentuated HE4 levels [8]. Interestingly, up regulation of HE4-V3 variation of HE4 is associated with a better clinical overall outcome.

The above examples illustrate the importance of assessing HE4 levels in other systemic malignancies and the need for further studies to fully evaluate the role of HE4 in tumor progression in these malignancies.

Conflict of interest The author declares no conflict of interest.

\section{References}

1. Azzam AZ, Hashad DI, Kamel NAF (2013) Evaluation of HE4 as an extrabiomarker to CA125 to improve detection of ovarian carcinoma: is it time for a step forward? Arch Gynecol Obstet. doi: 10.1007/s00404-013-2722-2

2. Kalogera E, Scholler N, Powless C et al (2012) Correlation of serum HE4 with tumor size and myometrial invasion in endometrial cancer. Gynecol Oncol 124:270-275

3. Angioli R, Plotti F, Capriglione S et al (2013) The role of novel biomarker HE4 in endometrial cancer: a case control prospective study. Tumour Biol 34:571-576

4. Mutz-Dehbalaie I, Egle D, Fessler S et al (2012) HE4 is an independent prognostic marker in endometrial cancer patients. Gynecol Oncol 126:186-191

5. Yamashita S, Tokuishi K, Moroga T et al (2012) Serum level of HE4 is closely associated with pulmonary adenocarcinoma progression. Tumour Biol 33:2365-2370

6. Iwahori K, Suzuki H, Kishi Y et al (2012) Serum HE4 as a diagnostic and prognostic marker for lung cancer. Tumour Biol 33:1141-1149

7. Elsammak MY, Attia A, Hassan HA, Zaytoun TM, Shorman M, Suleman M (2012) Evaluation of pleural fluid human epididymis 4 (HE4) as a marker of malignant pleural effusion. Tumour Biol 33:1701-1707

8. Tokuishi K, Yamashita S, Ohbo K, Kawahara K (2012) Splice variant HE4-V3 expression is associated with favorable prognosis in pulmonary adenocarcinoma. Tumour Biol 33:103-109 